The Spectrum of

Clean Energy Innovation

\title{
NREL Designs Promising New Oxides for Solar Cells
}

High-efficiency, thin-film solar cells require electrical contacts with high electrical conductivity, and the top contact must also have high optical transparency. This need is currently met by transparent conducting oxides (TCOs), which conduct electricity but are $90 \%$ transparent to visible light. Scientists at the National Renewable Energy Laboratory (NREL) have derived three key design principles for selecting promising materials for TCO contacts. NREL's application of these design principles has resulted in a 10,000-fold improvement in conductivity for one TCO material.

The upper TCO contact in a solar cell allows light to reach the absorber material below, which is the layer that generates free electrons via the photovoltaic effect. These electrons can then be conducted by the TCO to an external circuit, forming the negative terminal of the solar cell. TCOs used in this fashion are called $n$-type TCOs, because free electrons carry charges through the materials to make them conductive. TCOs can be enhanced by intentionally introducing other elements to a pure oxide, a technique known as "doping."

But finding and making so-called $p$-type TCOs—-where the charge is carried by positively charged "holes" created by the absence of electrons - has been a great challenge, despite much effort. Such $p$-type TCOs could be useful in a variety of electronic devices and could serve as positive terminals in solar cells, allowing new solar cell architectures. To find a suitable p-type TCO, the Center for Inverse Design (see sidebar on page 2) is pursuing an innovative approach that combines theory, experiment, and characterization: the perfect recipe for revolutionary results.

The starting point in NREL's approach is to determine a desired function for an improved material, in this case, a $p$-type TCO. Then, NREL scientists derive preliminary "design principles" for that material, which are a general set of requirements necessary to achieve that functionality. For TCOs, the design principles include the conditions for achieving transparency and conductivity, and for materials that naturally have a low electrical conductivity, a set of "doping rules" prescribe how to make those materials more conductive. Applying these preliminary design principles allows the selection of a first set of materials to examine more closely through high-throughput synthesis and characterization, and it may also lead to more refined design principles.

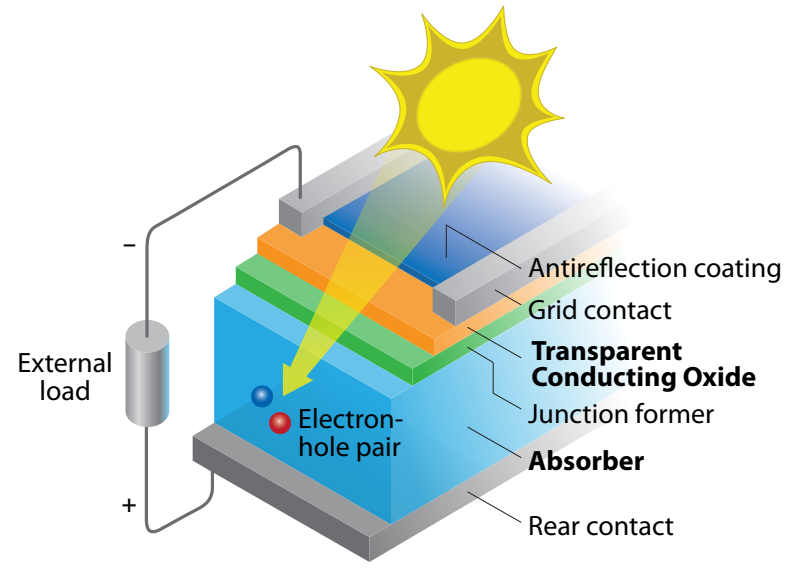

A transparent conducting oxide layer forms an upper electrical contact in a typical thin-film solar cell. Illustration by Al Hicks, NREL

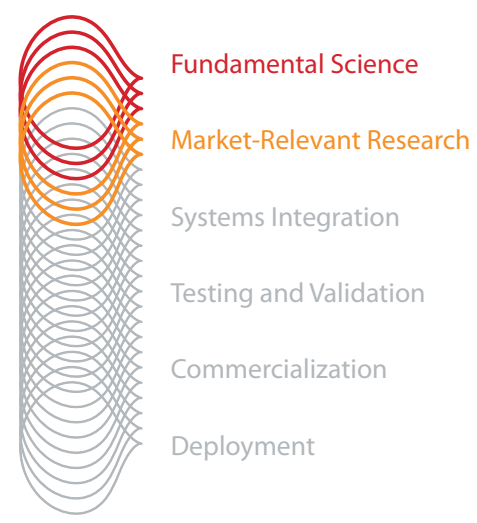

Through deep technical expertise and an unmatched breadth of capabilities, NREL leads an integrated approach across the spectrum of renewable energy innovation. From scientific discovery to accelerating market deployment, NREL works in partnership with private industry to drive the transformation of our nation's energy systems.

This case study illustrates NREL's innovations in Fundamental Science through Market-Relevant Research.

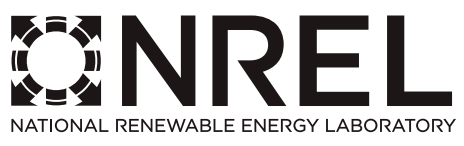

NREL is a national laboratory of the U.S. Department of Energy, Office of Energy Efficiency and Renewable Energy, operated by the Alliance for Sustainable Energy, LLC. 


\section{Considering Spinels}

In one study, NREL focused on finding improved p-type TCOs and chose to focus on crystalline spinels, because they exhibit some $p$-type behaviors. Spinels are a group of oxide minerals with the general chemical formula of $\mathrm{A}_{2} \mathrm{BO}_{4}$. This compound represents a crystal lattice of negative oxygen atoms (anions) and positive $A$ and $B$ atoms (cations). "A" cations can be Group III atoms (aluminum, titanium, iron, cobalt, or gallium) and "B" cations can be Group II atoms (magnesium, manganese, iron, nickel, or zinc) that occupy octahedral and tetrahedral sites, which are geometric locations between the regular lattice positions in the crystal. (a)

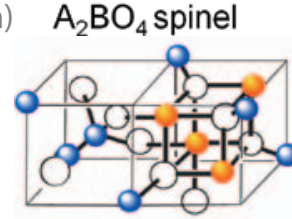

(b)

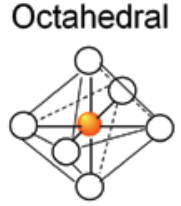

O Oxygen

A (a) Basic structure of the " $A$, ," $B$," and oxygen atoms in a spinel crystal $A_{2} B_{4}$. (b) In an octahedral ("8-triangular-faced") lattice site, one " $A$ " atom is bonded to six oxygen atoms; the right side of (a) shows an octahedral site. In a tetrahedral ("4-triangular-faced") lattice site, one " $B$ " atom is bonded to four oxygen atoms; the left side of (a) shows a tetrahedral site.

Using theory based on the fundamental laws of physics for a series of spinels, NREL scientists calculated the energy required to replace atoms in the crystal lattice with dopant atoms and then to create an electron-deficient hole in that lattice to serve as a carrier of a positive charge. Both energies must be low to allow the formation of positive charge-carrying holes that cause the spinel to become conductive. This work established a way to understand the difficulties of introducing $p$-type doping and sustaining holes in spinels.

\section{Refining Design Principles and Checking the Results}

From these calculations, NREL scientists were able to refine their design principles, deriving three new design principles for selecting candidate materials. First, find oxide groups immune to forming oxygen vacancies, this is, lattice positions missing an oxygen atom, which create unwanted $n$-type charge carriers. Some examples are standard $\mathrm{A}_{2} \mathrm{BO}_{4}$ spinels such as $\mathrm{Ga}_{2} \mathrm{MgO}_{4}, \mathrm{Al}_{2} \mathrm{MgO}_{4}$, and $\mathrm{Co}_{2} \mathrm{ZnO}_{4}$. Second, consider spinels likely to accommodate a high concentration of cation vacancies (where " $\mathrm{A}$ " or "B" atoms are missing in the regular lattice positions) or "anti-site" substitutions (where "A" is found at a typical "B" position, or vice versa). This cross-substitution of atoms tends to yield an excess of $p$-type charge carriers. Third, design systems or conditions free from potential compensating structures that oppose the beneficial results of doping. NREL's calculations on a large number of Group III-Group II spinels identified a particular class of spinels that have promising characteristics.

Scientists then performed experiments starting with $\mathrm{CO}_{2} \mathrm{ZnO}_{4}$ as a model system to validate, practice, and further refine these design rules for hole doping. The use of these design rules led to the deposition of $\mathrm{CO}_{2} \mathrm{NiO}_{4}$, which represents the end point of $\mathrm{CO}_{2} \mathrm{ZnO}_{4}$ doped with nickel in octahedral sites. With increasing nickel contentfrom $\mathrm{Co}_{2} \mathrm{ZnO}_{4}$ to $\mathrm{CO}_{2} \mathrm{NiO}_{4}$-electrical conductivity was boosted by a factor of 10,000.

\section{What's Next}

By using the inverse design approach, NREL scientists are creating a practical framework for future search and discovery of effective $p$-type oxide spinels based on design rules. But the search may extend beyond oxide spinels, because the " $\mathrm{X}^{\prime}$ in $\mathrm{A}_{2} \mathrm{BX}_{4}$ spinels does not need to be oxygen; it can also be sulfur, selenium, or tellurium, leading to a host of new candidates for contact material. The bottom line is that starting with the desired properties for a TCO, design principles can be developed to create materials with the potential to produce solar cells with optimal performance.

\section{NREL Targets the Grand Challenge of Materials by Design}

The Center for Inverse Design —an Energy Frontier Research Center funded by the U.S. Department of Energy and led by NREL — capitalizes on the laboratory's strong capabilities in theory, experiment, and characterization. Its mission is "to revolutionize the discovery of materials by developing an inverse design approach — using theory to guide experiments — with an initial focus on materials for solar energy conversion."

How do the inverse-design and conventional approaches differ? In the conventional approach, one chooses a material of known composition and structure, which often was discovered by accident to have useful properties. Then, retrospectively, one applies theory and targeted experiments to try to understand and explain the properties. The discovery of new technologically relevant materials is unpredictable and slow.

However, in NREl's inverse-design approach, this process is inverted by first defining the desired applicationspecific properties. Based on general scientific "design principles," NREL's scientists select a broad materials class within which the desired properties are likely to be found. They then employ high-throughput theoretical and experimental screening to identify the most promising candidate materials within this class. Finally, they perform in-depth studies by means of targeted theory, synthesis, and characterization to go the last mile, making the inverse design of materials a reality.

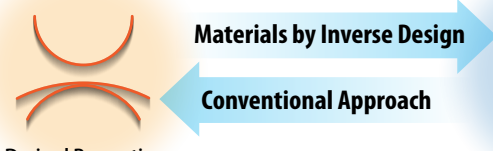

Desired Properties

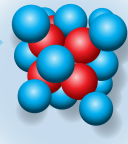
Materials

\section{National Renewable Energy Laboratory}

15013 Denver West Parkway

Golden, CO 80401

303-275-3000 • www.nrel.gov

NREL is a national laboratory of the U.S. Department of Energy, Office of Energy Efficiency and Renewable Energy, operated by the Alliance for Sustainable Energy, LLC.

NREL/FS-6A42-51557 • April 2012

Printed with a renewable-source ink on paper containing at least $50 \%$ wastepaper, including $10 \%$ post consumer waste.

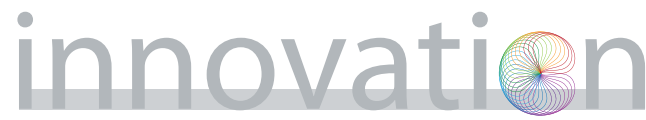

